\title{
Architecture of atrial musculature in humans
}

\author{
Ke Wang, Siew Yen Ho, Derek G Gibson, Robert H Anderson
}

\begin{abstract}
Objective-To investigate the gross arrangement of the principal muscular bundles of the two atria, and to suggest how it may contribute to contraction and spread of atrial excitation.

Design-A prospective analysis based on anatomical examination of adult human hearts.
\end{abstract}

Setting-A national heart and lung institute and a tertiary referral centre for cardiac disease.

Material-9 normal postmortem human hearts.

Methods-Dissection of atrial muscles with macrophotography.

Results-The atrial walls consist of circumferential and longitudinal muscular bundles, the former being arranged at the base of the atria with the latter predominating in the parietal walls. The muscular bundles in the right atrium are larger than those in the left. The main muscles forming the right atrial wall are the terminal crest and terminal pectinate muscles. The terminal crest, the most obvious muscle, is arranged longitudinally with its pectinate muscles connecting to the musculature of the atrioventricular vestibule. No structure resembling the terminal crest is seen in the left atrium. Instead the left atrial wall is composed of intermingled series of muscles, chief of these being the interatrial band and the septoatrial bundle. The former is arranged circumferentially at the atrial base, while the latter is mainly longitudinal. The wall of the right atrium is not of uniform thickness because of the presence of the terminal crest and its pectinate muscles on its internal surface. By contrast, the left atrial wall is much more uniform and its average thickness is greater than that of the right atrium. The rim of the oval fossa is the most important muscular structure on the septal surface and is formed by the infolded atrial walls. The other principal muscles of the atria attach to it, so that the rim provides mechanical support for overall movement of the atrial walls. Comparison of the gross arrangement of the atrial musculature with earlier echocardiographic measurements showed that this arrangement of the muscle explains movement of the atrioventricular ring and overall atrial contraction, and provides a suit- able substrate for preferential conduction.

Conclusion-The anatomical features of the atrial musculature explain the known facts concerning atrial contraction and preferential conduction.

(Br Heart f 1995;73:559-565)

Keywords: atrial anatomy; atrial muscles

Although there is a wealth of work devoted to the study of atrial function in the human heart, the topic of the atrial musculature has received scant attention from morphologists apart from the studies of Keith and Flack ${ }^{12}$ and Papez. ${ }^{3}$ In 1909, Flack ${ }^{4}$ emphasised the important role of the terminal pectinate muscles in atrial contraction and argued that "the function of the pectinate muscles of the atrium has been neglected." In 1920, Papez ${ }^{3}$ described and established more fully the arrangement of the atrial muscles of mammalian hearts, confirming the important role of the pectinate muscles. It is surprising, therefore, that there has been little further interest in these anatomical aspects despite much work being performed on measurements of atrial volume, area, and pressure. As the methods of investigating atrial function have developed, so has the need for precise anatomical information analysed in a clinical setting become more pressing. The purpose of this paper, therefore, was to reinvestigate the gross arrangement of the principal muscular bundles of the atria and to suggest how it may contribute to contraction and the spread of atrial excitation.

Materials and methods

Nine necropsied hearts were studied, all from patients who died of non-cardiac disease. The hearts were removed together with the proximal portions of the great vessels, promptly distended to a minimum degree with $10 \%$ formalin, and fixed for 1 week before study. The pericardial fat and epicardium were removed from the atrial mass, taking care not to disrupt the superficial muscle bundles. The arrangement of these bundles was then noted. Each atrial chamber was dissected and the endocardium was removed so as to disclose the arrangement of the internal musculature.

In describing our findings, we use the term "muscular bundle" or "band" in a macroscopical sense to refer to bundles that can be 
observed simply by removing the epicardium and endocardium. The relation between orientation of the muscle fibres and atrial contraction has been used to develop the concept of "circumferential" and "longitudinal" bundles within the atrial wall. The orientation of the circumferential fibres is parallel to the atrioventricular ring, while longitudinal fibres run parallel to the interatrial groove and at right angles to the atrioventricular junctions.

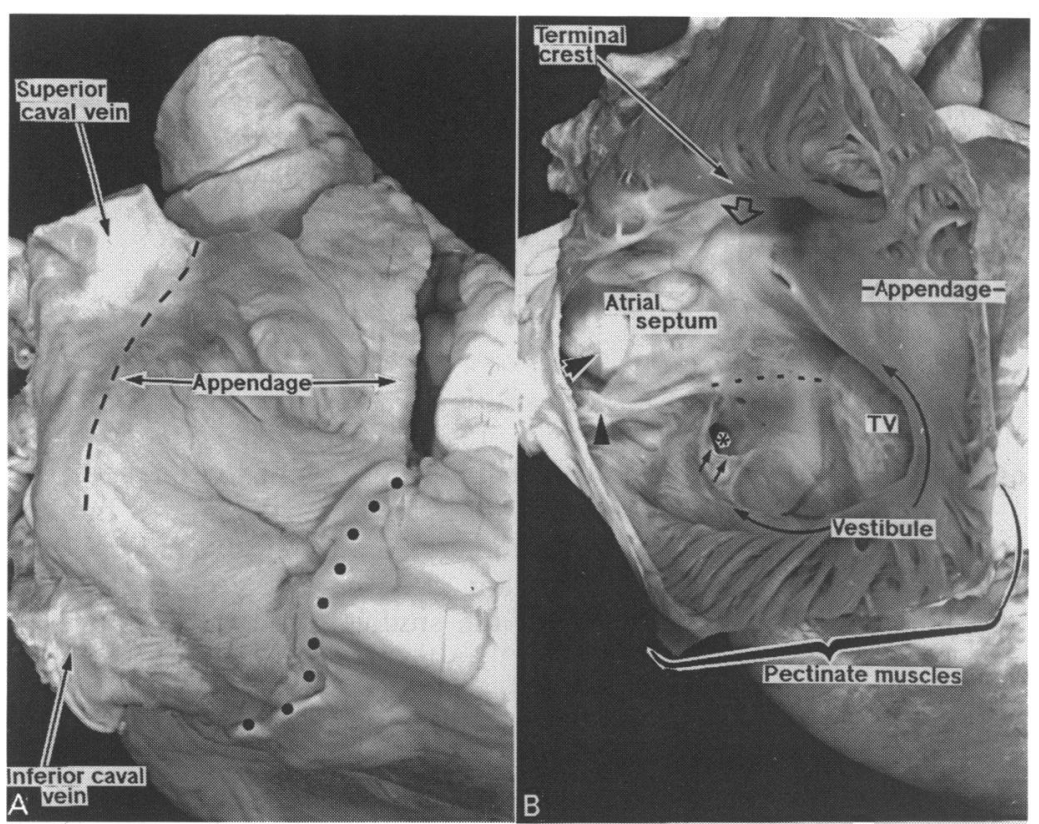

Figure 1 (A) External aspect of the right atrium showing a triangular shaped appendage. The terminal groove (broken line) marks the junction between the appendage and the venous sinus. The atrioventricular groove is indicated by the dotted line. (B) Internal aspect of the right atrium displayed through an incision made in the appendage. The incision has transected the pectinate muscles, which extend round the vestibule. The terminal crest (long arrow) passes in front, and to the right, of the entrance of the superior caval vein (open arrow). The openings of the inferior caval vein (solid arrow) and the coronary sinus $(*)$ are indicated. The tendon of Todaro (broken line) extends from the commissure between the Eustachian (arrow head) and Thebesian (double arrows) valves. The acute angle between the attachment of the tricuspid valve (TV) and the tendon forms the apex of the triangle of Koch.

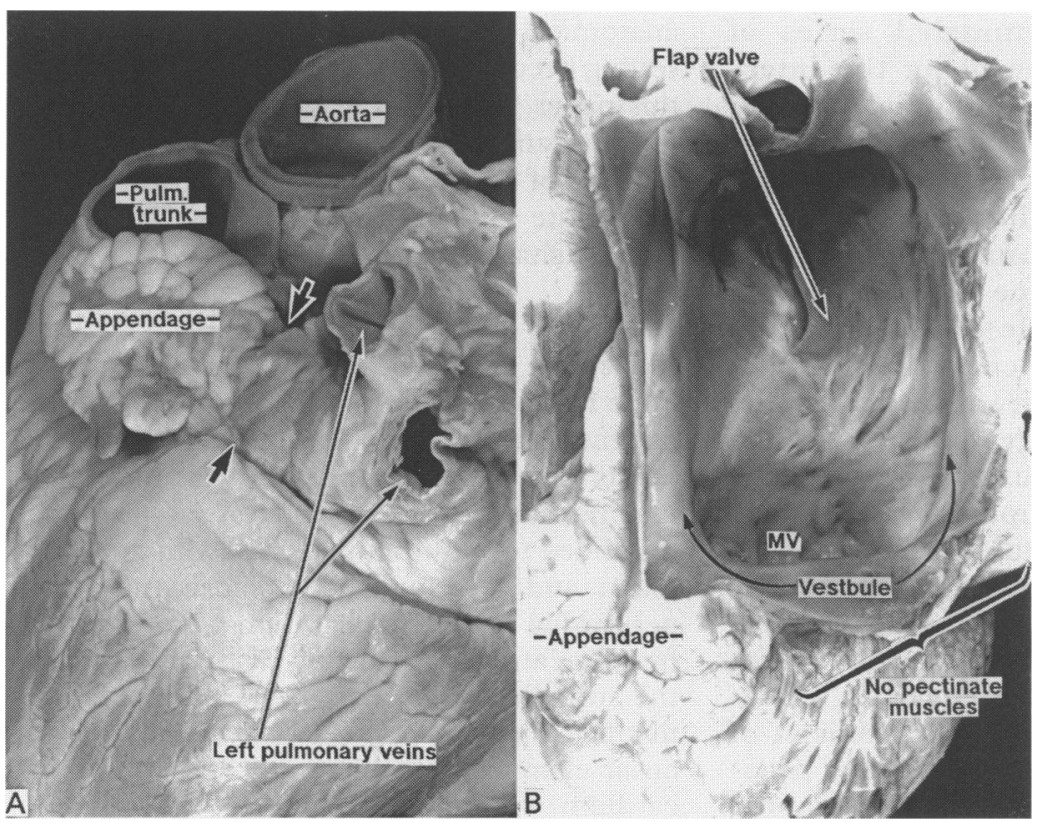

Figure 2 (A) External view of the left atrium showing the tubular appendage with a narrow junction (between arrows) to the pulmonary venous portion.

(B) Internal aspect showing the valve of the oval fossa on the septum. Pectinate muscles are confined to the appendage. The vestibule leading to the mitral valve (MV) is smooth. Pulm. trunk, pulmonary trunk.

\section{Results}

GROSS MORPHOLOGICAL STRUCTURE OF THE ATRIA

Each atrium has a venous component, an appendage and the vestibule of the atrioventricular valve, together with the septum and the adjacent walls between the chambers. In the morphologically right atrium (fig 1) the venous sinus is the posterior smooth walled component which receives the superior and inferior caval veins and the orifice of the coronary sinus. This is relatively small when viewed externally, extending between the terminal groove and the interatrial groove (Waterston's groove). The morphologically right appendage has a characteristically triangular shape, while internally it is lined by the pectinate muscles which originate from the terminal crest. The terminal crest is a prominent muscle on the internal surface which separates the pectinate lining of the appendage from the smooth walls of the venous sinus. Externally, the crest corresponds to the site of the terminal groove, in reality a line forming a surface landmark rather than a true gully. The vestibule of the tricuspid valve is the smooth muscular structure above the tricuspid ring, supporting and inserting into the leaflets of the tricuspid valve. In the right atrium, the pectinate muscles surround the entirety of the vestibule, reaching to the post-Eustachian sinus close to the crux.

In the morphologically left atrium (fig 2) the venous component, receiving the proximal parts of pulmonary veins, is considerably larger than the appendage. A terminal crest is lacking within the morphologically left atrium. The appendage itself is tubular, narrow, and hooked. The pectinate muscles themselves are less pronounced, being confined more or less within the appendage. The smooth walled vestibule of the left atrium, unrelated to the pectinate muscles, supports the leaflets of the mitral valve, the coronary sinus occupying the atrioventricular groove posteriorly.

The true atrial septum (fig 3) consists mainly of the flap valve of the oval fossa. It is only the immediate circumference of the fossa and its floor which is a muscular interatrial structure. The fossa has a well marked rim of muscle, nonetheless, and this constitutes an important muscular atrial component. The inferior part of the rim, known as the sinus septum, separates the orifice of the coronary sinus from that of the inferior caval vein. The tendon of Todaro, an important fibrous structure, courses through this area from the zone of fusion between the fibromuscular valve guarding the orifice of the inferior caval vein (the Eustachian valve) and the orifice of the coronary sinus (the Thebesian valve). The sinus septum is continuous anteriorly with the atrioventricular muscular septum, the atrial component of which forms the surface of the triangle of Koch.

\section{MUSCULAR ARCHITECTURE OF THE ATRIA} Atrial walls

The atrial walls consist of intricately intermingled muscular bundles orientated 
Figure 3 Right atrium showing the limit of the oval fossa (broken line) on the septal aspect. Orifices of the inferior caval vein (closed arrow) and coronary sinus $(*)$ are guarded by the Eustachian (arrow head) and Thebesian (double arrows) valves respectively. [ $\Longrightarrow]$, orifice of the superior caval vein; inf. caval vein inferior caval vein.

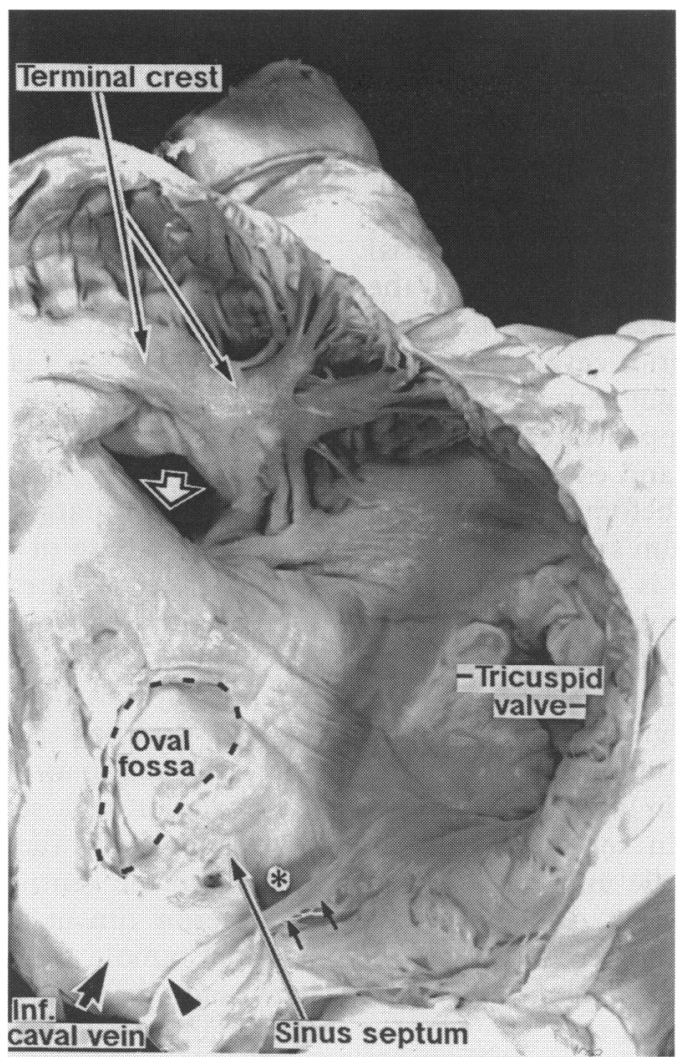

circumferentially and longitudinally. The circumferential bundles are most distinct at the base of the atria, while longitudinal bundles are mostly found in the internal walls. The atrial musculatures at the atrioventricular junctions are separated completely from ventricular mass by the fibrofatty planes of atrioventricular insulation.

\section{Right atrium}

The largest and strongest muscular bundle in the right atrium is the longitudinal muscular ridge known as the terminal crest (crista terminalis) (fig 4(A)). It originates from the anterior aspect of the septum, swings in front of the orifice of the superior caval vein, and continues downwards on the right of the orifices of both caval veins, diminishing in size and merging inferiorly into the subEustachian sinus near the orifice of the coronary sinus. Along its course, it gives rise to about $15-20$ pectinate muscles, almost parallel muscular ridges which extend anterolaterally and posteriorly in the wall of the right atrium (fig $4(\mathrm{~A})$ ). One or two of the upper pectinate muscles are very large (1-3 $\mathrm{mm}$ in diameter) and extend at right angles into the appendage. The most prominant is often termed the septum spurium. The rest of the pectinate muscles divide into numerous branches in the lateral or posterior wall of the right atrium, incline towards the vestibule of the tricuspid valve, and directly attach to the tricuspid ring at right angles. The lowermost pectinate muscle encircles the orifice of the coronary sinus and joins the sinus septum.

There are two circumferential bundles on the internal and external surfaces of the right atrium which encircle together the base of the atrium and form the vestibular muscles. The internal circumferential bundle originates from the anterior aspect of the septum, encircles the vestibule, and extends posteriorly (fig 4(B)). Throughout its course, its lower margin gives rise to the thin fibres attaching to the fibrous tricuspid ring. Its upper margin receives perpendicularly the attachment of the pectinate muscles from the terminal crest. atrium opened through an incision which passes parallel to the right coronary artery to the tip of the appendage and then through the terminal crest $(\mathrm{O}-\mathrm{O})$ into the superior caval vein. Pectinate muscles arise along the length of the terminal crest $(++)$ toward the coronary sinus $\left({ }^{\star}\right)$. The Eustachian valve in this heart is particularly prominent.

(B) Endocardium of the right atrium has been removed to reveal the internal circumferential bundle (arrows) encircling the vestibule. The pectinate muscles (lines) are perpendicular to the circumferential bundle. + , terminal crest; sup. caval vein, superior caval vein.

(C) Atria viewed from behind showing

Waterston's groove (broken line) and the intercaval bundle after removal of epicardium. The terminal groove is not visible as a line of depression. Rt. upper pulm. vein, right upper pulmonary vein; $r$. lower pulm. vein, right lower pulmonary vein; sup. caval vein; superior caval vein; inf. caval vein, inferior caval vein.

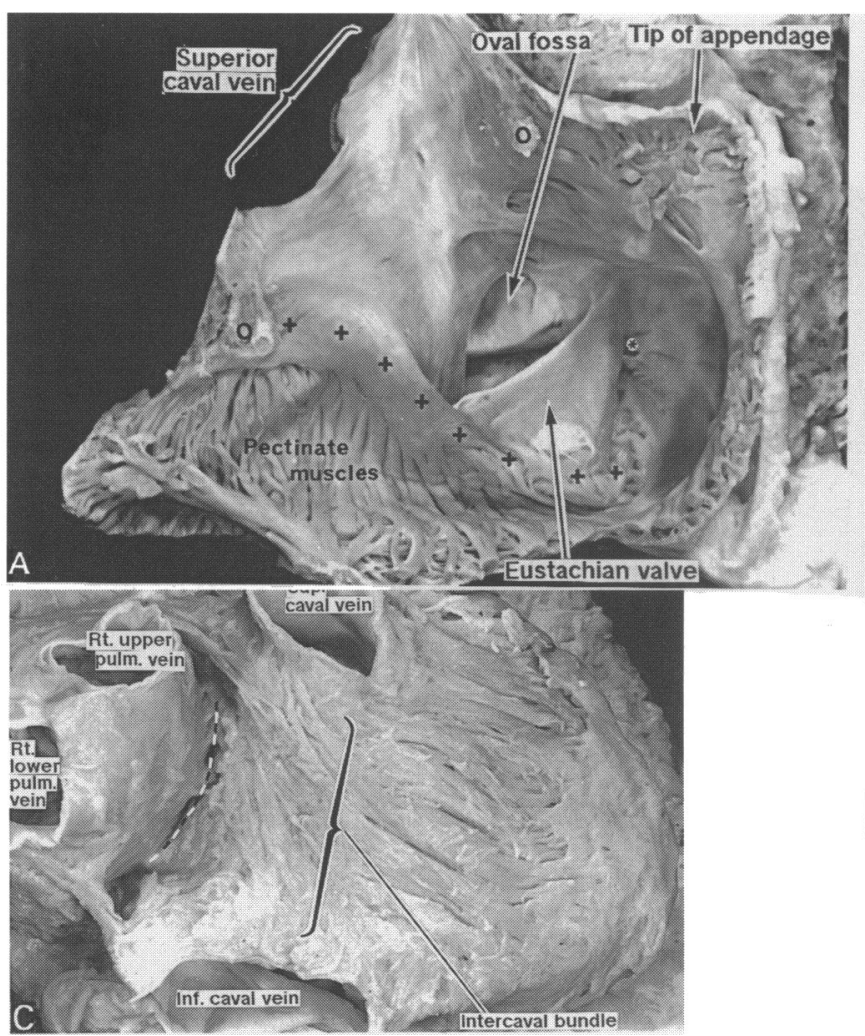


Papez ${ }^{3}$ called this bundle "the right anterior crest". Not as strong as the terminal crest, it is a flattened bundle which is distinct in the anterior aspect but scarcely visible in the posterior aspect. We have called it the right annular bundle. The external circumferential bundle is the right extremity of the interatrial band which lies on the anterior surface of the right atrium. This band originates at the right of the orifice of the superior caval vein or the atriocaval junction, its right extremity passing to the front of the right appendage, dividing to encircle the appendage, and extending posteriorly in feeble fashion before finally joining the lower part of Waterston's groove.

The intercaval bundle is the major muscular bundle of the venous sinus, covering the surface of the sinus between Waterston's groove and the terminal groove (fig $4(\mathrm{C})$ ). Crossing the terminal groove, this bundle becomes the external bundle of the right atrium, spreading as a thin layer and overlying the pectinate muscles. In some cases, however, the intercaval bundle and the external bundle are often so weakly developed that they cannot be defined with certainty.

\section{Left atrium}

The circumferential bundles in the left atrium are larger and stronger than those in the right atrium, again lying mostly on the vestibule. The principal circumferential bundle is the left extremity of the interatrial band (known also as Bachmann's bundle). This is best seen on the external surface of the left atrium (fig 5(A)). It extends from the right of the orifice of the superior caval vein or the atriocaval junction and crosses this vessel and the anterior wall of the left atrium transversely until it approaches the left appendage, where it divides into upper and lower branches which encircle the narrow mouth of the appendage. The upper branch extends from the appendage in front of the entrance of the orifices of the left pulmonary vein to the lateral wall. Its lower branch extends inferiorly to the atrial base, encircles the vestibule, and attaches to the mitral ring or the area of aortic mitral continuity of the fibrous skeleton. These two branches interdigitate with other circumferential bundles on the lateral wall, continue posteriorly, and insert into the lower part of Waterston's groove.

The left atrial longitudinal bundle, "the left septoatrial bundle", is a flattened muscle seen internally (fig $5(\mathrm{~B})$ ). It originates on the anteroinferior margin of the septum, passes to the anterior base of the atrium, and attaches to the leaflet of mitral ring. It then breaks up into longitudinal and circumferential bundles. The longitudinal bundle, the main branch, extends upwards and backwards into the superior and posterior wall between the right and left pulmonary veins. It then spreads on the posterior wall, some fibres extending into the vestibule and the mitral ring, while others insert into the central fibrous body as part of the inferior rim of the septum. The circumferential bundle continues transversely to the left and backwards, intermingling with Bachmann's bundle on the lateral wall.

The muscle bundle of the sinus component in the left atrium, "the septopulmonary bundle", is larger than the comparable bundle of the right atrium. It originates from Waterston's groove and passes upwards to the anterior and superior wall behind Bachmann's bundle, where it ramifies to form as many as six branches. The largest fascicle becomes a circumferential bundle which continues to the left and intermingles with Bachmann's bundle on the lateral wall. The remaining branches, some very feeble, make various changes in orientation and cover the venous component almost longitudinally from the front to behind.

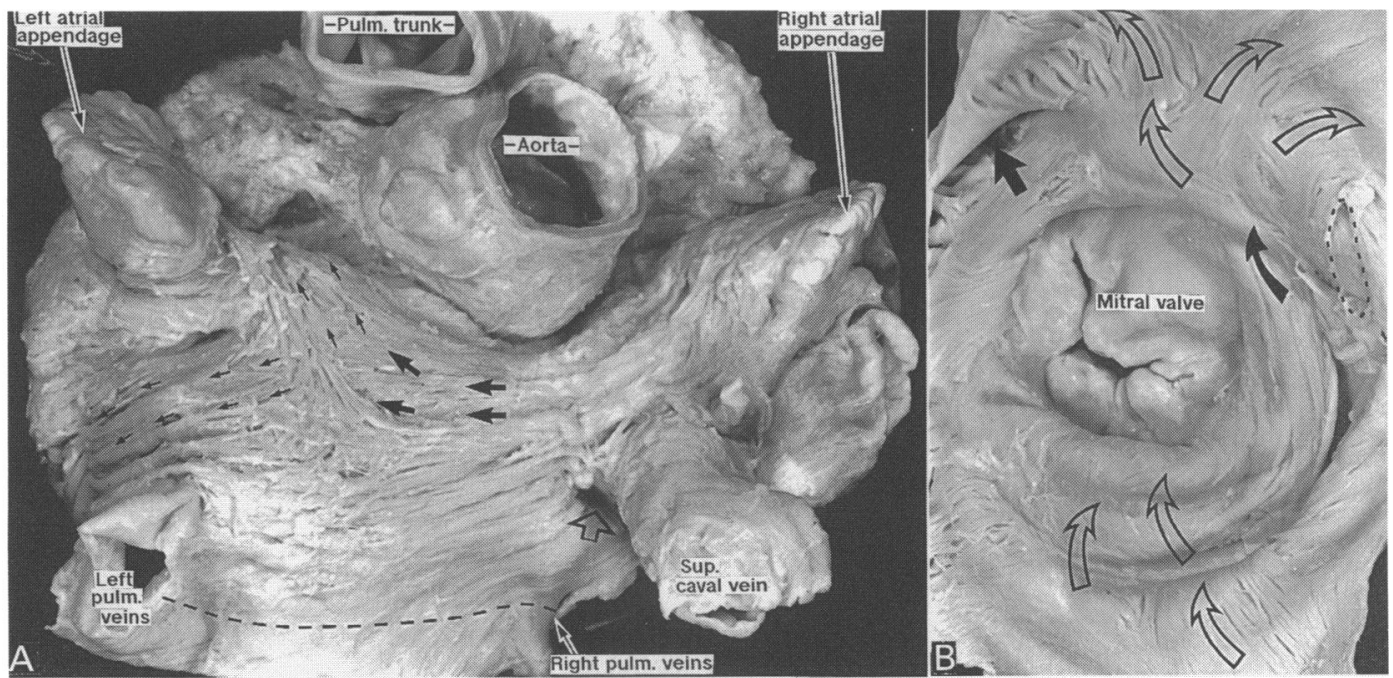

Figure 5 (A) Atria viewed from above after removal of epicardium. Interatrial band (large closed arrow) divides into upper and lower branches (small closed arrows). [C], Waterston's groove; - - - , line of incision made to display the inside of the left atrium as shown in $(B)$.

(B) Septoatrial bundle (curved closed arrow) passes anteriorly to join with the longitudinal bundle (curved open arrows) which run between the left and right pulmonary veins. Pulm. trunk, pulmonary trunk; left pulm. veins, left pulmonary veins; right pulm. veins, right pulmonary veins; sup. caval vein, superior caval vein. 
Figure 6 (A) Atria viewed from behind to show deep infolding of the atrial wall which makes up the anterior and superior rims of the oval fossa.

(B) Further dissection reveals the infolded rim (broken line) and the flap valve of the oval fossa. *, orifice of the coronary sinus; sup. caval vein, superior caval vein, inf caval vein, inferior caval vein.

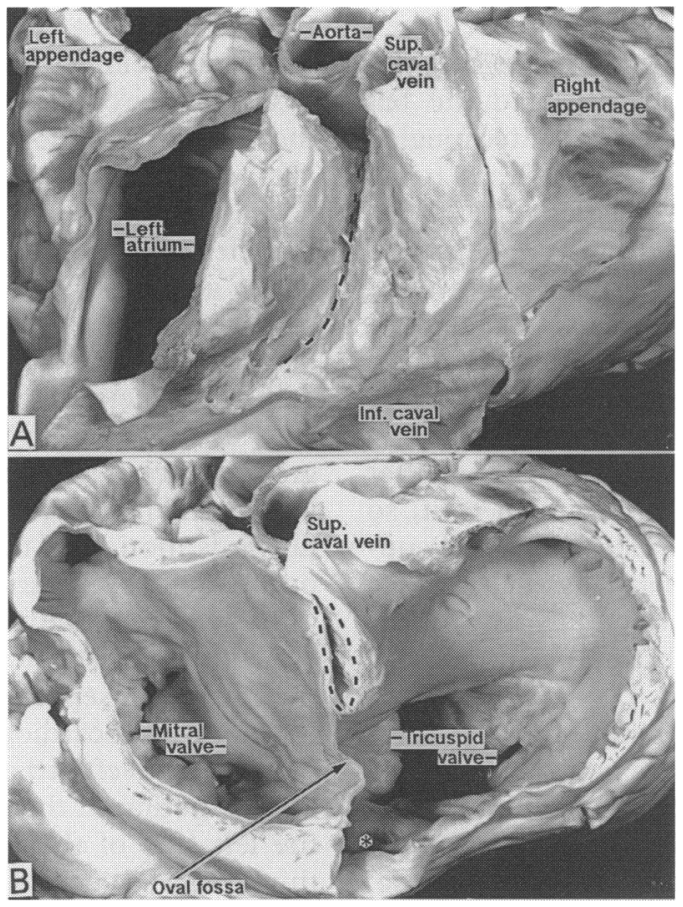

Atrial septum

The rim of the oval fossa, being best seen in the right atrium, is an important muscular structure, but is formed by the infolded atrial walls. The other principal bundles of the atria attach to it. The superior part of the rim is produced by an infolding of the muscular fibres between the venous sinus of the right atrium and the right pulmonary veins of the left atrium. The intercaval bundle of the right atrium together with the septopulmonary bundle of the left atrium originate from this part of the rim and extend toward the right or

Figure 7 Internal view of the right atrium after removal of endocardium showing the apparently extensive nature of the atrial septum comprising the oval fossa (broken line) and its muscular rim. $\leftrightarrow$ line of incision made into the superior caval vein $(S)$ to produce fig 6(B);

* coronary sinus;... attachment of tricuspid valve; sup. rim, superior rim; inf. rim, inferior rim.

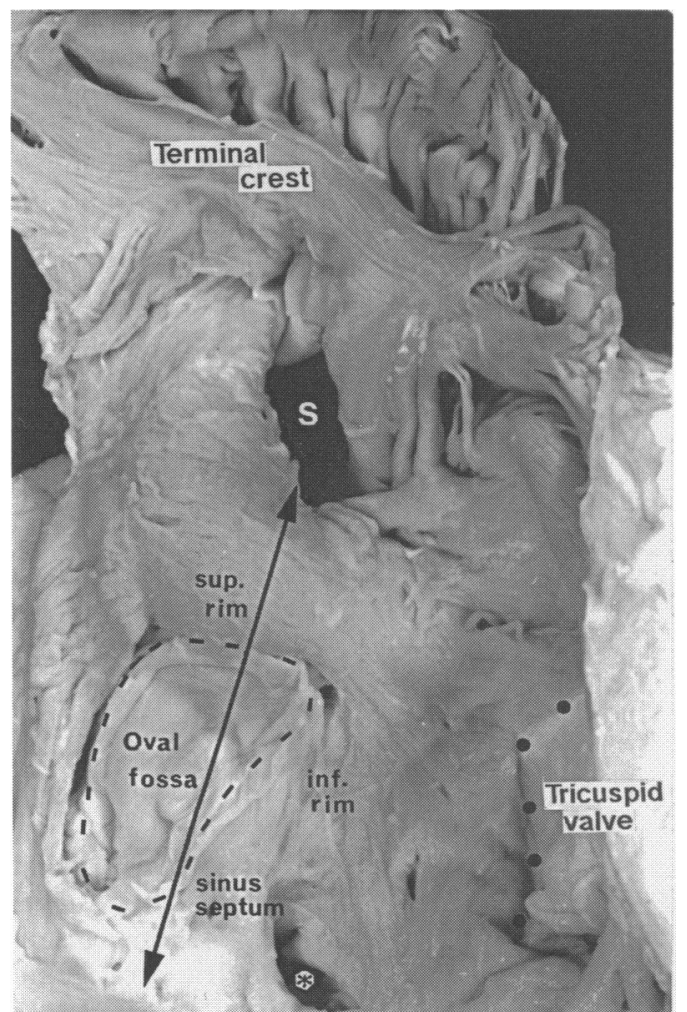

left sinus components. The anterior part of the rim is an extensive folding of atrial musculature in the vertically orientated interatrial groove (fig 6). The terminal crest, the right annular bundle of the right atrium, and the septoatrial bundle of the left atrium originate here. The inferior part of the rim overlies the central fibrous body and continues backwards as the sinus septum, the muscular structure which separates the orifice of the coronary sinus from that of the inferior caval vein (fig 7).

\section{Wall thickness}

The right atrial wall is not of uniform thickness because the terminal crest and its pectinate muscles constitute a considerable proportion of the right atrial wall. The thickest region is at the top of the terminal groove, varying between 5 and $8 \mathrm{~mm}$. The right atrial wall becomes exceedingly thin towards the vestibule. The thinnest part is at the anterior or posterior aspect of the vestibule, being about $2 \mathrm{~mm}$. The left atrial wall is much more uniform. The thickest region is at the anterior wall, about ( $4-5 \mathrm{~mm}$ ), while the thinnest part is the posterior or anterior aspect of the vestibule (about $3 \mathrm{~mm}$ ). On average, nevertheless, the left atrium has thicker walls than the right atrium.

\section{Discussion}

It is axiomatic that an understanding of the complex architecture of the atrial musculature should improve understanding of its activation and contraction. In $1920 \mathrm{Papez}^{3}$ studied in detail the musculature of the right and the left atria, as well as the venous sinus, in human, bovine, and canine hearts. He did not, however, consider the relation between the orientation of atrial muscles and contraction, so he simply used the concept of internal and external muscles rather than taking into account the circumferential and longitudinal orientation of the fibres.

Our observations confirm that the atrial walls consist of intermingling circumferential and longitudinal muscular bundles (fig 8). The circumferential muscles surround the base of the atria, mirroring the arrangement of ventricular circumferential muscle fibres which surround the ventricular bases. The circumferential atrial muscles are most distinct anteriorly, where they cross transversely to the posterior base of the atria as a thin, incomplete layer. The main longitudinal muscular bundles are internal or deep fibres which pass over the roof of each atrium to the corresponding atrioventricular ring.

There are distinct differences in the arrangement of the muscular bundles between the two atria in terms of their individual identity. Those in the morphologically right atrium are stronger and larger than those in the left, although the left atrial wall is thicker. The main muscles forming the right atrial wall are the terminal crest and terminal pectinate muscles, these reinforcing internally the relatively thin parietal wall of the appendage. No 
structure resembling the terminal crest is seen in the left atrium, and the pectinate muscles are much less pronounced. Instead the left atrial wall is composed of an intermingled series of muscles, chief of these being Bachmann's bundle and the septoatrial bundle. These left atrial structures are relatively inconspicuous as surface landmarks, however, compared with the terminal crest and its pectinate muscles in the right atrium. The rim of the oval fossa is another important muscular structure on the interatrial septum, being formed by the infolded atrial walls. The other principal muscles of the atria attach to it, so that the rim provides overall mechanical support for movement of the atrial walls.

The initial purpose of our study was to elucidate the anatomical substrate for atrial contraction. Flack ${ }^{4}$ emphasised the function of the pectinate muscles. He argued that the terminal crest was a fixed point through the venous mesocardium, from which the pectinate muscles originated, terminating in the musculature of the atrioventricular ring. When the pectinate muscles contract, therefore, they are drawn towards this fulcrum, the ventricle being drawn simultaneously. The atrioventricular ring thus moves towards the venous base of the heart. This movement at one stroke empties the atrium and fills the ventricle during atrial systole. Keith ${ }^{2}$ demonstrated this function of the pectinate muscles by injecting warm wax into the atrium and showed that the pectinate muscles must shorten to nearly half their diastolic length during atrial systole. Papez ${ }^{3}$ also thought that mass contraction of the atrium was caused by contraction of the pectinate muscles and their connections with the rim of the oval fossa through the terminal crest.

In a previous study with $M$ mode echocardiography ${ }^{5}$ we confirmed that the atrioventricular ring moved up towards the atria during atrial systole, the tricuspid ring moving further than the mitral ring. We now suggest that these changes reflect the striking differences in

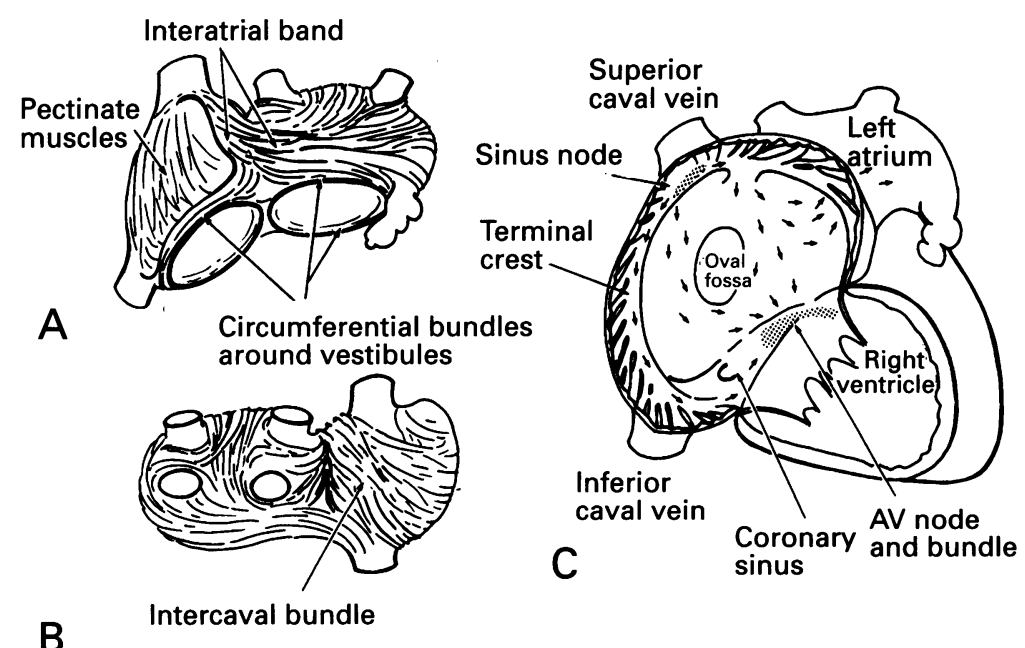

Figure 8 Diagrammatic representation of the $(A)$ front and $(B)$ the back views of the overall arrangement of the atrial musculature after removal of epicardium. (C) Right atrium and landmarks to the triangle of Koch. $\rightarrow$, major muscle bundles through which preferential conduction passes from the sinus node to the atrioventricular node and to the left atrium. $A V$, atrioventricular. the arrangement of the right and left atrial muscular architecture.

Overall, circumferential muscles seem to be the same size as longitudinal muscles in the left atrium. The circumferential muscle (Bachmann's bundle) at the anterior wall of the left atrium, very close to the posterior wall of the aorta, is the strongest. Circumferential muscle, therefore, may be the cause of the backward motion of the aortic posterior wall and the reduction in the anteroposterior atrial axis documented by $M$ mode echocardiography during left atrial systole. By contrast, longitudinal muscle is likely to produce upwards motion of the mitral ring which also occurs during left atrial systole.

In previous studies reported by ourselves ${ }^{6}$ and other investigators ${ }^{7}$ long axis motion of the interatrial septum during atrial systole on $M$ mode echocardiography was observed to be similar to left atrial motion, being reflected by the motion of the central fibrous body and the left side of the atrioventricular ring. We think that it may also be related to the constitution of the fibrous skeleton. The fibrous skeleton consists of the central fibrous body and the left fibrous trigone. ${ }^{8}$ The former, formed by the union of the right fibrous trigone and the membranous septum, lies just under the inferior rim of the oval fossa and receives the attachment of muscular fibres from the rim. The latter is composed of fibrous tissue between the leaflets of the mitral and aortic valves, and gives attachment to muscle fibres of the left atrium. Attachment of muscle fibres to these skeletal structures explains why motion of the interatrial septum and left atrium is similar but not necessarily identical during atrial systole.

Our study also provides anatomical evidence concerning the substrates for preferential conduction. In the early 1900 s Keith and Flack $^{1}$ considered the terminal crest as the principal primary pathway for the excitation process in the right atrium, while Bachmann ${ }^{9}$ proposed the interatrial band as the primary pathway for conduction from right to left atrium. Keith and Flack ${ }^{1}$ designated the interatrial band as "a constant band passing from the sinus musculature to the vestibule of the left atrium". Many studies ${ }^{1011}$ have since proved that the mode of conduction of impulses within the atria is through pathways of preferential conduction rather than purely radial spread, and that this conduction occurs along well developed muscular bundles, albeit that the bundles are not insulated electrically from the rest of the atrial myocardium. Rather, it is the geometry of the major atrial muscular bundles that is thought to provide the faster routes for excitation from the sinus node and for propagation to all parts of the atria. ${ }^{10}{ }^{11}$ Our studies do not permit us to comment on whether preferential conduction might be a consequence of special electrophysiological properties of the cells in these bundles. They suggest that normal activation of the atriums can readily be explained by the geometric arrangement of atrial musculature without needing to involve any hypothetical 
specialised atrial pathways. First, the margins of the great veins and the oval fossa together with the prominent and thick terminal crest constitute the shortest and most prominent conduction pathways from the sinus node to the atrioventricular node. ${ }^{12}$ The holes in the atrium (caval veins, coronary sinus, oval fossa) direct the impulse from the sinus node to take precisely these routes to reach the right atrium, the atrioventricular node, and the left atrium (fig 8). Second, the muscle fibres in the well developed muscular bundles are arranged in parallel fashion. It has been well established that the propagation in a direction parallel to a fibre bundle is faster than propagation in a direction at right angles to the bundle (anisotropic conduction). ${ }^{13-15}$ On this basis, therefore, the excitation wave from the sinus node might be expected to spread first to the right atrium along the terminal crest and the right annular bundle, then to the interatrial septum along the anterior rim of the oval fossa, and finally to the left atrium along two pathways, one via the atriocaval junction and Bachmann's bundle, and the other along the rim of the oval fossa. ${ }^{1011} 1617$

This work was supported by the British Heart Foundation and the Royal Brompton Hospital Special Cardiac Fund. We thank Dr Jessica Mann for providing some of the specimens.

1 Keith A, Flack $M$. The form and nature of the muscula connections between the primary divisions of the verteconnections between the primary divisions
brate heart. $\mathcal{F}$ Anat Physiol 1907;41:172-89.

2 Keith A. An account of the structures concerned in the production of the venous pulse. F Anat Physiol 1907; 42:1-25.

3 Papez JW. Heart musculature of the atria. Am $\mathcal{f}$ Anat 1920-21;27:255-77.

4 Flack M. The heart. In: Hill L, ed. Further advances in physiology. London: Edward Arnold, 1909:34-71.

5 Jones CJH, Song GJ, Gibson DG. An echocardiographic assessment of atrial mechanical behaviour. Br Heart $f$ assessment of

6 Henein MY, Priestley K, Davarashvili T, Buller N, Gibson DG. Early changes in left ventricular subendocardial DG. Early changes in left ventricular subendocardial
function after successful coronary angioplasty. $\mathrm{Br}$ Heart function after succes

7 Keren G, Sonnenblick EH, Lejemtel TH. Mitral annulus motion. Relation to pulmonary venous and transmitral flows in normal subjects and in patients with dilated cardiomyopathy. Circulation 1989;78:621-9.

8 Anderson RH, Becker AE. The heart. London: Gower Medical Publishing, 1992;1:31-2.

9 Bachmann G. The inter-auricular time interval. $A m f$ Physiol 1916;41:309-20.

10 Spach MS, King TD, Barr RC, Boaz DE, Morrow MN, Herman-Giddens S. Electrical potential distribution surrounding the atria during depolarization and repolarization in the dog. Circ Res 1969;23:857-73.

11 Spach MS, Lieberman M, Scott JG, Barr RC, Johnson EA, Kootsey JM. Excitation sequences of the atrial sep$\mathrm{EA}$, Kootsey JM. Excitation sequences of the atrial sep-
tum and the AV node in isolated hearts of the dog and tum and the AV node in isolated

12 Anderson RH, Ho SY, Smith A, Becker AE. The internodal atrial myocardium. Anat Rec 1981;201:75-82.

13 Clerc L. Directional differences of impulse spread in trabecular muscle from mammalian heart. $f$ Physiol 1976; 255:335-46.

14 Roberts DE, Hersh LT, Scher AM. Influence of cardiac fibre orientation on wave front voltage, conduction velocity and tissue resistivity in the dog. Circ Res 1979; 44:701-12.

15 Spach MS, Miller WT, Dolbert PC, Kootsey JM, Sommer JR, Mosher CE Jr. The functional role of structural complexities in the propagation of depolarization in the atrium of the dog. Cardiac conduction disturbances due to discontinuities of effective axial resistivity. Circ Res 1982;50:175-91.

16 Janse MJ, Anderson RH, Van Capelle FJL, Durrer D. A combined electrophysiological and anatomical study of the human fetal heart. Am Heart $₹$ 1976;91:556-62.

17 Spach MS, Dolber PC, Sommer JR. Discontinuous propagation: an hypothesis based on known cardiac structure complexities. Int $\mathcal{F}$ Cardiol 1985;7:167-74. 